\title{
Estratégias para o ensino da prática baseada em evidências na formação de enfermeiros: revisão integrativa
}

\section{Estrategias para la enseñanza de la práctica basada en la evidencia en la educación de enfermería: una revisión integradora}

\section{Strategies for teaching evidence-based practice in nurse education: integrative review}

\author{
Recebido: 20/05/2017 \\ Aprovado: 15/12/2017 \\ Publicado: 07/05/2018
}

Fernanda Carolina Camargo ${ }^{1}$ Helena Hemiko Iwamoto ${ }^{2}$ Gilberto de Araújo Pereira ${ }^{3}$ Regiane Máximo de Souza ${ }^{4}$ Luan Augusto Alves Garcia ${ }^{5}$ Damiana Aparecida Trindade Monteiro6 Sabrina Karla de Souza Cubas $^{7}$ Gabriela França Rosinha ${ }^{8}$

Este estudo tem como objetivo identificar estratégias para o ensino da Prática Baseada em Evidências nos diferentes níveis de formação dos enfermeiros. Trata-se de uma revisão integrativa, cuja busca ocorreu nas bases PubMed, CINAHL e LILACS. Foram incluídos estudos em inglês, português e espanhol, publicados em periódicos de enfermagem, no período de janeiro de 2007 a outubro de 2016. Foram identificadas 68 referências, e atenderam aos critérios de inclusão 13 estudos primários. Estratégias identificadas para o ensino foram a mediação do processo ensino-aprendizagem no cenário da prática e as estratégias propiciadas pelas tecnologias de informação e comunicação, além da reorientação da matriz educacional. 0 fortalecimento da integração ensinoserviço, a formação de enfermeiros Mestres e Doutores para atuarem como consultores para a implementação da PBE nas organizações de saúde, e a reflexão sobre a atuação docente apresentaram-se como eminentes contribuições para a formação do enfermeiro de práticas avançadas. As evidências identificadas podem apoiar alternativas de ensino para o fortalecimento da formação do enfermeiro ancorado em conhecimento científico consubstanciado; também, elas podem apoiar a discussão do tema no âmbito na América Latina e Caribe, onde os estudos sobre o tema são incipientes.

Descritores: Enfermagem baseada em evidências; Pesquisa em enfermagem; Docentes de enfermagem; Educação em enfermagem.

This study aims to identify strategies for the teaching of evidence based practice in the different levels of nursing training. It is an integrative review carried out in the PubMed, CINAHL and LILACS databases. Studies in English, Portuguese and Spanish, published in nursing journals from January 2007 to October 2016 were included. Sixty-eight references were identified, and thirteen primary studies met the inclusion criteria. The teaching strategies identified were the mediation of the teaching-learning process in the practice scenario and strategies resulting from information and communication technologies, in addition to the reorientation of the educational matrix. The strengthening of the teaching-service integration, the training of Master and PhD nurses to act as consultants for the implementation of the EBP in health organizations, and the reflection on the teaching performance were presented as eminent contributions to the training of nurses of advanced practices. The evidences identified can support teaching alternatives for strengthening the training of nurses anchored in scientific knowledge. They can also support the discussion of the theme in the scope in Latin America and the Caribbean, where studies on this topic are incipient.

Descriptors: Evidence-based nursing; Nursing research; Faculty nursing; Education nursing.

Este estudio tiene el objetivo de identificar estrategias para la enseñanza de la Práctica Basada en Evidencias en los diferentes niveles de formación de los enfermeros. Se trata de una revisión integradora, cuya búsqueda ocurrió en las bas es PubMed, CINAHL y LILACS. Fueron incluidos estudios en inglés, portugués y español, publicados en periódicos de enfermería, en el periodo de en ero de 2007 a octubre de 2016. Fueron identificadas 68 referencias, y atendieron a los criterios de inclusión 13 estudios primarios. Estrategias identificadas para la enseñanza fueron la mediación del proceso enseñanza-aprendizaje en el escenario de la práctica y las estrategias propiciadas por las tecnologías de información y comunicación además de la reorientación de la matriz educacional. El fortalecimiento de la integración enseñanza-servicio, la formación de enfermeros Maestros y Doctores para actuar como consultores para la implementación de la PBE en las organizaciones de salud, y la reflexión sobre la actuación docente se presentaron como eminentes contribuciones para la formación del enfermero de prácticas avanzadas. Las evidencias identificadas pueden apoyar alternativas de enseñanza para el fortalecimiento de la formación del enfermero anclado en conocimiento científico consubstanciado; también ellas pueden apoyar la discusión del tema en el ámbito en América Latina y del Caribe, donde los estudios sobre el tema son incipientes.

Descriptores: Enfermería basada en la evidencia; Investigación en enfermería; Docentes de enfermería; Educación en Enfermería.

1. Enfermeira. Mestre e Doutora em Atenção à Saúde. Epidemiologista Clínica do Setor de Pesquisa e Inovação Tecnológica (SPIT) da Gerência de Ensino e Pesquisa (GEP) do Hospital de Clínicas da Universidade Federal do Triângulo Mineiro (HC-UFTM), Uberaba, MG, Brasil. ORCID: 0000-0002-1048-960X E-mail: fernandaccamargo@yahoo.com.br

2. Enfermeira. Doutora e Mestre em Enfermagem. Professora Associada do Curso de Enfermagem da UFTM. Uberaba, MG, Brasil. 0RCID: 0000-0003-1125-4252 E-mail: helena.iwamoto@gmail.com

3. Estatístico. Doutor em Estatística. Docente do Curso de Enfermagem e do Programa de Pós Graduação no Mestrado Profissional em Inovação Tecnológica da UFTM. Uberaba, MG, Brasil. ORCID: 0000-0002-9149-6368 E-mail: pereira_gilberto@yahoo.com.br

4. Bacharel em Matemática. Doutora em Engenharia de Produção. Docente junto ao Departamento de Engenharia de Produção e ao Programa de Pós Graduação em Engenharia de Produção da Universidade Estadual Paulista Júlio de Mesquita Filho (UNESP), Bauru, SP, Brasil. ORCID: 0000-0002-4695-2678 E-mail: regiane@feb.unesp.br

5. Enfermeiro. Especialista em Saúde do Trabalhador. Especialista em Gestão dos Serviços em Ergonomia. Especialista em Saúde do Adulto, na modalidade Residência Integrada e Multiprofissional. Mestre em Atenção à Saúde. Doutorando do Programa de Pós Graduação em Atenção à Saúde (PPGAS) - UFTM, Uberaba, MG, Brasil. ORCID: 0000-0003-0984-2688 E-mail: luangarciatpc@yahoo.com.br

6. Enfermeira. Mestranda do PPGAS - UFTM, Uberaba, MG, Brasil. ORCID: 0000-0002-6740-7687 E-mail: damianaatm@hotmail.com

7. Discente do curso de graduação em Enfermagem da UFTM, Uberaba, MG, Brasil. ORCID: 0000-0002-3112-9515 E-mail: sasa.souza1501@hotmail.com

8. Discente do curso de graduação em Enfermagem da UFTM, Uberaba, MG, Brasil. ORCID: 0000-0002-1061-5805 E-mail: gabrielarosinha@hotmail.com 


\section{INTRODUÇÃO}

A Prática Baseada em Evidências (PBE) na Enfermagem apoia a tomada de decisões clínicas e gerenciais quanto à aplicação de resultados de pesquisas científicas robustas para a atuação cotidiana. De forma geral, os princípios para o cuidado de Enfermagem pautados em evidências científicas emergem no cenário assistencial frente à necessidade de um melhor controle dos custos, de ampliar a efetividade da assistência mediante limitações econômicas, como também de garantir a qualidade dos serviços de saúde prestados e a segurança do paciente ${ }^{1-3}$.

É esperado que o enfermeiro tenha conhecimentos e habilidades para a avaliação crítica de pesquisas, identificação da melhor evidência científica, e para combinar esses resultados com sua expertise clínica, as preferências do paciente e os recursos disponíveis nos contextos dos serviços de saúde ${ }^{1-4}$. Mesmos sendo uma concepção normatizada há mais de duas décadas pelas associações de Enfermagem nos países anglosaxões - como a American Nursing Association e a American Association Nursing College - essa perspectiva ainda se encontra incipiente no que diz respeito aos enfermeiros dos países da América Latina e Caribe $^{3,4}$.

Todavia, a implementação da PBE na Enfermagem apresenta-se como um desafio mundial1-5, por enfrentar alguns dilemas relacionados à natureza das pesquisas em Enfermagem, ao apoio dos serviços de saúde para a implementação da PBE, e à competência dos enfermeiros para atuarem com essa prática ${ }^{3,6}$. As pesquisas em Enfermagem, por serem uma produção de conhecimento que incorpora suas próprias teorias e paradigmas sócio-humanísticos, em sua maioria são empreendidas por meio de abordagens não exclusivas quantitativas ou ensaios clínicos ${ }^{2-9}$. As organizações de saúde, muitas vezes, não disponibilizam acesso rápido e fácil à literatura científica atual especializada em Enfermagem, como também inexiste a proteção de horas na carga de trabalho para o consumo de pesquisas e de normativas que apoiem ou institucionalizem a incorporação de inovações no processo de trabalho das equipes de Enfermagem 1-3,5,10.

A limitação na competência individual dos enfermeiros para o exercício da PBE apresenta-se como um dos fatores mais críticos neste cenário. Entende-se que o curso da ação de incorporar uma evidência científica ao cuidado envolve atitudes além da apresentação de intervenções alternativas, seguras cientificamente, a pessoas, famílias e comunidades assistidas ${ }^{1-3,6,7}$. Trata-se de uma ação complexa, que envolve o julgamento crítico do enfermeiro; conhecimento de diferentes delineamentos de pesquisa; aceitação da nova intervenção pelas pessoas assistidas considerando suas crenças e subjetividades; mobilização da equipe de enfermagem para modificar hábitos do exercício profissional; opinião de outras categorias implicadas diretamente na assistência, como os médicos; e identificação da disponibilidade de recursos na instituição para a sustentação da mudança ${ }^{1-3,6,7,10}$. Mediante este panorama, são requeridas habilidades diferenciadas ao enfermeiro na implementação de sua atuação guiada pela PBE. Emergem questionamentos quanto à formação habitual dos enfermeiros, se ela tem possibilitado aproximações teóricopráticas às dimensões que tangenciam a complexidade da PBE para esta categoria profissional.

Contudo, a melhoria da qualificação do profissional de Enfermagem em sua competência técnico-científica tem sido tema de debate no contexto contemporâneo, no que diz respeito à formação de recursos humanos em saúde 2,4,11-13. O estímulo ao consumo de resultados de pesquisas e a aproximação com o desenvolvimento das mesmas é atividade a ser fomentada desde a graduação ${ }^{11,12}$. Acrescenta-se a esta realidade a necessidade eminente de induzir, na América Latina e Caribe, a formação diferenciada do profissional de Enfermagem para enfrentamento das complexas demandas atuais em saúde, que emergem em uma sociedade em constante transformação $0^{4,13}$.

Idealmente, o profissional formado desta maneira traria o cuidado de saúde o 
mais próximo possível de onde as pessoas vivem e trabalham, guiado por conhecimentos especializados e pensamento crítico decisório para escolher e desenvolver produtos e processos em saúde, baseado em práticas cientificamente bem fundamentadas e socialmente aceitáveis,2,411-13. Também é esperado deste profissional de Enfermagem, de perfil inovador, que seja um agente motivado a desempenhar a liderança transformacional ${ }^{5,10-12}$. 0 Conselho Internacional de Enfermeiros define que para o estabelecimento deste perfil profissional o enfermeiro de prática avançada -, a formação educacional deve ir além do escopo tradicional, e aponta a necessidade de ampliar o grau de formação, acrescentando níveis além do bacharelado como pósgraduação nos níveis Mestrado e Doutorado ${ }^{13,14}$.

Mediante os desafios contemporâneos que requerem a inclusão de conteúdos diferenciados na formação, e a consubstanciação entre o ensino e a vivência de cuidados de Enfermagem baseados em métodos, tecnologias e práticas cientificamente fundamentadas, questionase: Quais estratégias podem ser empreendidas para o ensino da PBE nos diferentes níveis de formação da Enfermagem? Observa-se na literatura contemporânea uma escassez de estudos que reflitam sobre esta temática pela perspectiva de trabalho do docente, sobretudo, pela incipiência do tema nos cenários de formação ${ }^{15,16}$. Ante o exposto, este estudo tem como objetivo identificar estratégias para o ensino da Prática Baseada em Evidências nos diferentes níveis de formação dos enfermeiros.

\section{MÉTODO}

Trata-se de uma revisão integrativa (RI), recurso metodológico para reunir, avaliar e sintetizar evidencias científicas na Enfermagem ${ }^{17-19}$. Este método permite a inclusão para análise de estudos primários com diferentes abordagens metodológicas e delineamentos de pesquisa, e não apenas ensaios clínicos randomizados como seria proposto em uma revisão sistemática ${ }^{17,18}$. A RI permite a avaliação crítica das evidências encontradas e a caracterização do estado de conhecimento do assunto de interesse ${ }^{17-19}$. A presente RI foi conduzida nas seguintes etapas: desenvolvimento da questão de pesquisa para o desenvolvimento da revisão; busca na literatura dos estudos primários; extração dos dados; avaliação dos estudos primários; interpretação dos resultados e apresentação da revisão ${ }^{18}$. A questão de pesquisa delimitada para o desenvolvimento da revisão foi: Quais são as estratégias utilizadas para o ensino da PBE nos diferentes níveis de formação dos enfermeiros? Foi utilizada para a construção da questão de estudo a estratégia $\mathrm{PICO}^{20}$, sendo $\mathrm{P}$ a população (enfermeiros em diferentes níveis de formação), e I a intervenção ou área de interesse (as estratégias de ensino da prática baseada em evidências); os elementos C (comparação entre intervenção ou grupo) e 0 (desfecho) não foram empregados.

As bases de dados foram a PubMed (National Library of Medicine National Institutes of Health), CINAHL (Cumulative Index to Nursing and Allied Health Literature) e LILACS (Ciências da Saúde da América Latina e Caribe). Os descritores controlados utilizados em inglês para PubMed e CINAHL foram [Evidence Based Nursing], [Evidencebased practice], [Associate degree nursing]; e em português na LILACS: [Enfermagem Baseada em Evidências], [Prática Clínica Baseada em Evidências], [Educação em Enfermagem]. Como descritores não controlados em inglês para PubMed e CINAHL empregou-se; [Nursing Education, Associate], [Associate Nursing Education], [Associate Nursing Educations], [Education, Associate Nursing], [Educations, Associate Nursing], [Nursing Educations, Associate], em português na LILACS: [Ensino em Enfermagem]. Os descritores foram combinados de diferentes maneiras para garantir ampla busca de estudos primários. As buscas ocorreram em outubro de 2016.

Para realizar a análise foram incluídos estudos em inglês, português e espanhol, indexados em periódicos de enfermagem, no período de janeiro de 2007 a outubro de 2016, disponíveis como texto completo e que apresentassem a descrição de estratégias 
para o ensino da PBE. Foram excluídos os estudos de revisão, cartas editoriais, estudos realizados em unidade ou clínica específicas. Quanto às populações investigadas foram excluídos os estudos primários que tiveram a participação exclusiva de enfermeiros assistenciais ou gerenciais. A primeira seleção dos estudos primários foi por meio da leitura, por pares independentes, dos títulos e resumos das diferentes listagens de referências identificadas nas bases de dados. Em seguida, as pesquisas foram minuciosamente lidas na íntegra, também por pares independentes. A partir desta etapa houve a eliminação dos estudos que não apresentassem a descrição da estratégia educativa empreendida para o ensino da PBE. A busca manual por meio da leitura das referências dos estudos primários incluídos também foi empregada, sendo inseridos os estudos que contemplassem os critérios de inclusão delimitados na revisão.

A extração dos dados dos estudos primários foi baseada em um instrumento utilizado na literatura brasileira ${ }^{21}$, e considerou os seguintes aspectos: dados da publicação (autores, ano, periódico e local da publicação), objetivo, delineamento do estudo, resultados e conclusão. Os estudos primários foram classificados conforme: nível 1, meta-análise de múltiplos estudos controlados; nível 2, estudo individual com desenho experimental; nível 3, estudo com desenho quase experimental como estudo sem randomização com grupo único pré e pós-teste, séries temporais ou caso-controle; nível 4, estudo com desenho nãoexperimental como pesquisa descritiva correlacional e qualitativa ou estudos de caso; nível 5, relatórios de casos ou dados obtidos de forma sistemática, de qualidade verificável, ou dados de avaliação de programas; nível 6 , opinião de autoridades respeitáveis baseada na competência clínica ou opinião de comitês de especialistas, o que inclui interpretações de informações baseadas em pesquisas, incluindo opiniões reguladoras ou legais ${ }^{22}$. Os achados dos estudos primários foram organizados em categorias, conforme estratégias empreendidas para o ensino da PBE.

Quanto aos aspectos éticos, as informações específicas extraídas dos artigos foram acessadas por meio de bancos de dados, não necessitando de autorização para utilizá-las por se tratarem de material pertencente ao domínio público.

\section{RESULTADOS}

A busca resultou em 68 artigos, sendo apenas três repetidos entre os diferentes recursos informacionais. 13 estudos primários atenderam aos critérios de inclusão, nenhum dos quais foi incluído pela busca manual (Tabela 1).

Tabela 1. Bases consultadas conforme quantidade de referências recuperadas, selecionadas e estudos primários incluídos. Uberaba, Minas Gerais, Brasil, 2016.

\begin{tabular}{lccc}
\hline Bases & $\begin{array}{c}\text { Referências } \\
\text { Recuperadas }\end{array}$ & $\begin{array}{c}\text { Referências Selecionadas por Títulos e } \\
\text { Resumos }\end{array}$ & $\begin{array}{c}\text { Estudos Primários } \\
\text { Incluídos }\end{array}$ \\
\hline PubMed & 43 & 15 & 05 \\
CINAHL & 47 & 10 & 08 \\
Lilacs & 04 & 01 & 00 \\
Total & 94 & 26 & 13 \\
\hline
\end{tabular}

Quanto aos periódicos, Nurse Education Today Journal $(\mathrm{n}=3)$ e Journal of Professional Nursing $(\mathrm{n}=2)$ foram os que apresentaram maior número de artigos (Quadro 1).

Através da síntese das estratégias identificadas para o ensino da PBE nos diferentes níveis de formação dos enfermeiros, foram elaboradas três categorias: estratégias mediadoras do processo ensino-aprendizagem, estratégias propiciadas pelas tecnologias de informação e comunicação e estratégias para reorientação da matriz educacional. Das estratégias mediadoras do processo ensinoaprendizagem, (intervenções a serem implementadas no cotidiano da atividade de ensino) observou-se: discussão de casos clínicos, projetos entre academia e cenário de 
prática, leitura análise e revisão crítica de artigos (Quadro 2).

Quadro 1. Caracterização dos estudos primários incluídos quanto a autores, ano de publicação, periódicos, delineamento do estudo, nível de evidência e localidade da publicação. Uberaba, Minas Gerais, Brasil, 2016.

\begin{tabular}{|c|c|c|c|c|}
\hline Autor (es) & Periódico (ano) & Delineamento & Nível & Localidade \\
\hline Stone \& Rowles ${ }^{23}$ & $\begin{array}{l}\text { Journal of Nursing Management } \\
(2007)\end{array}$ & $\begin{array}{l}\text { Exploratório } \\
\text { descritivo }\end{array}$ & 4 & Estados Unidos \\
\hline $\begin{array}{ll}\text { Adams } & \& \\
\text { MacCarthy } & \end{array}$ & $\begin{array}{l}\text { The Journal of School Nursing } \\
(2007)\end{array}$ & Estudo teórico & 6 & Estados Unidos \\
\hline $\begin{array}{l}\text { McNett, Fusilero \& } \\
\text { Mion }^{25}\end{array}$ & Nurse Leader (2009) & Estudo teórico & 6 & Estados Unidos \\
\hline Gray $^{26}$ & Nurse Education Today (2009) & $\begin{array}{l}\text { Longitudinal } \\
\text { descritivo }\end{array}$ & 4 & Estados Unidos \\
\hline Stiffler \& Cullen ${ }^{27}$ & $\begin{array}{l}\text { Journal of Professional Nursing } \\
(2010)\end{array}$ & Relato de experiência & 5 & Estados Unidos \\
\hline Larmon \& Varner ${ }^{28}$ & $\begin{array}{l}\text { Teaching and Learning in Nursing } \\
\text { (2011) }\end{array}$ & Relato de experiência & 5 & Estados Unidos \\
\hline $\begin{array}{l}\text { Janke, Pesut \& } \\
\text { Erbacker }{ }^{29}\end{array}$ & Nurse Education Today (2012) & Relato de experiência & 5 & Canadá \\
\hline Sortedahl ${ }^{30}$ & $\begin{array}{l}\text { Worldviews on Evidence } \\
\text { Nursing (2012) }\end{array}$ & Relato de experiência & 5 & Estados Unidos \\
\hline Zhang et $a l^{31}$ & Nurse Education Today (2012) & Estudo piloto & 4 & China \\
\hline Buchholz et al ${ }^{32}$ & $\begin{array}{l}\text { Journal of the American Association } \\
\text { of Nurse Practitioners (2013) }\end{array}$ & Estudo teórico & 6 & Estados Unidos \\
\hline $\begin{array}{l}\text { Debruyn, Ochoa- } \\
\text { Marín \& Semenic }{ }^{33}\end{array}$ & $\begin{array}{l}\text { Investigación Educación Enfermería } \\
\text { (2014) }\end{array}$ & Qualitativo descritivo & 4 & Colômbia \\
\hline $\begin{array}{l}\text { Hunker, Gazza \& } \\
\text { Shellenbarger }\end{array}$ & $\begin{array}{l}\begin{array}{l}\text { Journal of Professional Nursing } \\
(2014)\end{array} \\
\end{array}$ & Estudo teórico & 6 & Estados Unidos \\
\hline $\begin{array}{l}\text { André, Aune \& } \\
\text { Braend }^{35}\end{array}$ & Nurse Education in Practice (2016) & Relato de experiência & 5 & Noruega \\
\hline
\end{tabular}

Quadro 2. Estratégias identificadas para o ensino da prática baseada em evidências nos diferentes níveis de formação dos enfermeiros, correspondentes à categoria estratégias mediadoras do processo ensino-aprendizagem. Uberaba, Minas Gerais, Brasil, 2016.

\begin{tabular}{|c|c|}
\hline Estratégias mediadoras do processo ensino-aprendizagem & Autores \\
\hline $\begin{array}{l}\text { Discutir durante os estágios casos clínicos e gerenciais que possibilitema a } \\
\text { identificação de uma questão clínica e a busca de evidências para } \\
\text { respondê-la }\end{array}$ & $\begin{array}{l}\text { Stone \& Rowles }{ }^{23} \text {; Zhang et al }{ }^{31} \text {; } \\
\text { André, Aune \& Braend }{ }^{35}\end{array}$ \\
\hline $\begin{array}{l}\text { Elaborar projetos colaborativos de pesquisa e intervenção entre } \\
\text { estudantes e enfermeiros do cenário de prática }\end{array}$ & $\begin{array}{l}\text { Stone \& Rowles }{ }^{23} \text {; } \\
\text { Zhang et al }{ }^{31} ; \text { André, Aune \& } \\
\text { Braend } 35\end{array}$ \\
\hline $\begin{array}{l}\text { Elaborar roteiros para orientar os estudantes sobre a integração de } \\
\text { evidências científicas na prática assistencial }\end{array}$ & Adams \& MacCarthy 24 \\
\hline $\begin{array}{l}\text { Orientar o desenvolvimento de habilidades sobre a escrita de artigos } \\
\text { científicos e a apresentação oral de resultados de pesquisa }\end{array}$ & Hunker, Gazza \& Shellenbarger ${ }^{34}$ \\
\hline $\begin{array}{l}\text { Realizar atividades que contenham a leitura, análise e revisão crítica de } \\
\text { artigos científicos de diferentes delineamentos metodológicos }\end{array}$ & Larmon \&Varner ${ }^{28}$ \\
\hline
\end{tabular}

\section{Quanto as estratégias propiciadas} pelas tecnologias de informação e comunicação, àquelas que se relacionam à veiculação do ambiente online como motriz para o fomento do ensino da PBE - a facilitação aos espaços virtuais depositários de pesquisas, e a divulgação de redes colaborativas em pesquisa foram os principais aspectos apresentados (Quadro 3).
Já, as estratégias para reorientação da matriz educacional, foram aquelas que abordaram conceitos a serem incluídos nos currículos de Enfermagem, como a oferta de disciplinas complementares, a atuação de enfermeiros doutores como consultores e o fortalecimento de parcerias entre a acadêmica e cenários de atuação no sentido 
de ampliar a formação de Enfermeiros de Práticas Avançadas (Quadro 4).

Quadro 3. Estratégias identificadas para o ensino da prática baseada em evidências nos diferentes níveis de formação dos enfermeiros, correspondentes à categoria estratégias propiciadas pelas tecnologias de informação e comunicação. Uberaba, Minas Gerais, Brasil, 2016.

\begin{tabular}{|l|l|}
\hline \multicolumn{1}{|c|}{$\begin{array}{c}\text { Estratégias propiciadas pelas Tecnologias } \\
\text { de Informação e Comunicação }\end{array}$} & \multicolumn{1}{c|}{ Autores } \\
\hline $\begin{array}{l}\text { Facilitar o acesso às pesquisas dos estudantes de enfermagem, por meio, } \\
\text { por exemplo, de recursos informacionais e bases de dados }\end{array}$ & $\begin{array}{l}\text { Sortedahl30; Debruyn, Ochoa- } \\
\text { Marín \& Semenic }\end{array}$ \\
\hline $\begin{array}{l}\text { Divulgar grupos de pesquisas e redes colaborativas de produção de } \\
\text { conhecimento e inovações aos estudantes de enfermagem }\end{array}$ & $\begin{array}{l}\text { Sortedahl30; Debruyn, Ochoa- } \\
\text { Marín \& Semenic }{ }^{33}\end{array}$ \\
\hline $\begin{array}{l}\text { Organizar espaços virtuais colaborativos entre as escolas de enfermagem } \\
\text { e/ou universidades para que possam difundir inovações, ampliar a } \\
\text { utilização da PBE e discutir as dificuldades para o seu ensino }\end{array}$ & Sortedahl30 \\
\hline
\end{tabular}

Quadro 4. Estratégias identificadas para o ensino da prática baseada em evidências nos diferentes níveis de formação dos enfermeiros, correspondentes à categoria estratégias para reorientação da matriz educacional. Uberaba, Minas Gerais, Brasil, 2016.

\begin{tabular}{|c|c|}
\hline Estratégias para orientação da matriz educacional & Autores \\
\hline $\begin{array}{l}\text { Ofertar cursos ou disciplinas complementares que dê aos estudantes a } \\
\text { oportunidade de experimentar a geração e incorporação de resultados de } \\
\text { pesquisa na prática }\end{array}$ & $\begin{array}{l}\text { Stiffler \& Cullen }{ }^{27} \text {; Janke, Pesut \& } \\
\text { Erbacker }{ }^{29} \text {; Zhang et al }{ }^{31} \text {; André, } \\
\text { Aune \& Braend }{ }^{35}\end{array}$ \\
\hline $\begin{array}{l}\text { Formar enfermeiros doutores para que atuem como consultores no } \\
\text { desenvolvimento de pesquisas, avaliação e incorporação de seus } \\
\text { resultados na prática assistencial, em hospitais ou outros serviços de } \\
\text { saúde }\end{array}$ & $\begin{array}{l}\text { McNett, Fusilero \& } \quad \text { Mion } \\
\text { Buch ; }\end{array}$ \\
\hline $\begin{array}{l}\text { Integrar no currículo tópicos que abordem as etapas para implementação } \\
\text { da PBE }\end{array}$ & 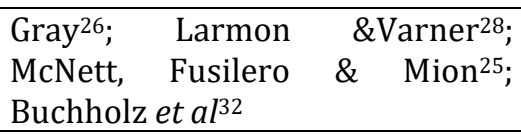 \\
\hline $\begin{array}{l}\text { Fortalecer as parcerias entre escolas de enfermagem/universidades e } \\
\text { serviços de saúde }\end{array}$ & $\begin{array}{l}\text { Gray }{ }^{26} \text {; Zhang et al }{ }^{31} \text {; André, Aune } \\
\text { \& Braend }{ }^{35} ; \text { Debruyn, Ochoa- } \\
\text { Marín \& Semenic }{ }^{32}\end{array}$ \\
\hline $\begin{array}{l}\text { Orientar a matriz pedagógica para a Prática Avançada em Enfermagem, a } \\
\text { fim de desenvolver o julgamento crítico, e o estímulo a inovações para } \\
\text { solução de problemas, por meio de um modelo colaborativo, de ciências } \\
\text { translacionais e sensível aos aspectos socioculturais }\end{array}$ & $\begin{array}{l}\text { Stiffler \& Cullen }{ }^{27} \text {; Janke, Pesut \& } \\
\text { Erbacker } 29 ; \text { Zhang et al }{ }^{31} \text {; André, } \\
\text { Aune \& Braend }{ }^{35}\end{array}$ \\
\hline
\end{tabular}

\section{DISCUSSÃO}

Estudos indicaram que a atuação dos estudantes de Enfermagem nos cenários de prática é veiculadora da aproximação das evidências científicas junto aos enfermeiros gerentes e equipes de enfermagem das unidades assistenciais ${ }^{23,26,28}$, em especial, quando os estudantes desenvolvem projetos de pesquisa que têm como interface intervenções que procuram solucionar problemas identificados nestes cenários.

De forma geral, os projetos apresentaram boa aceitação pelas equipes de enfermagem, e a maior contribuição identificada foi a aproximação dos trabalhadores com informações atualizadas sobre investigações científicas correspondentes a temas que se relacionavam com problemas clínicos vivenciados nas unidades assistenciais ${ }^{23,26,28}$.

0 fortalecimento da integração ensino-serviço foi apontado como iniciativa facilitadora para a maior viabilização do ensino e da vivência da PBE entre os estudantes de Enfermagem ${ }^{30,33}$. Entretanto, esta aproximação deve ser orientada por uma matriz curricular que discuta a PBE de forma transversal ao longo das disciplinas em que se baseia a formação do bacharel em Enfermagem ${ }^{26-28}$.

Tal matriz curricular, também, deve oferecer atividades e cursos complementares específicos, incluindo estratégias educativas online $^{30}$, para que os alunos possam vivenciar essa realidade nos cenários assistenciais, mesmo que por simulação ${ }^{28}$. 
Etapas, guias e componentes para o ensino da incorporação de evidências científicas na prática foram delineadas em outros estudos $24,27,28,30$. Os componentes orientadores destes guias de ensino da PBE abordaram e exemplificaram como elaborar a proposta de intervenção, definir as palavraschave para a extração de pesquisas, identificar riscos frente à implementação da nova intervenção e avaliar o sucesso alcançado na implementação deste novo processo entre os estudantes de Enfermagem $24,27,28,30$.

Os estudos primários analisados abordaram a essencialidade da integração e ensino fortalecido da PBE na pós-graduação de Enfermagem, sendo esperado, sobremaneira, que os enfermeiros cuja formação contemple esses elementos se tornem profissionais com domínio ampliado e consistente em sua competência para orientar a implementação da PBE nos cenários assistenciais $25,29,31,32,34,35$. Frente a essas proposições, cada vez mais torna-se necessárias investigações que verifiquem como estratégias mediadoras do processo ensino-aprendizagem e a utilização das tecnologias de informação e comunicação tem sido empreendidas para a formação à PBE em diferentes localidades.

Mediante $\mathrm{o}$ advento da internet, espaços virtuais colaborativos entre as escolas de enfermagem e universidades apresentam-se como potencialidades para ensino da PBE em maior escala - com acesso difundido ao maior número de enfermeiros, sem barreiras geográficas.

No contexto da América Latina e Caribe, em especial por serem cenários com incipiente produção na temática, infere-se que a utilização das tecnologias de informação e comunicação apresentam-se como recursos viabilizadores ao incremento dessa abordagem. Haja vista que o acesso oportuno ao conhecimento especializado contribui, sobremaneira, para desdobramentos na formação - tendo como imagem o alcance da formação da Prática Avançada de Enfermagem.

$\mathrm{Na}$ formação no nível de mestrado e doutorado se deve consolidar o alcance do perfil profissional do enfermeiro de práticas avançadas $25,29,31,32,34,35$. Os resultados apresentados colaboram para destacar o protagonismo que Universidades e Cursos de Enfermagem têm na formação diferenciada desses profissionais, sendo uma de suas missões permanentes aperfeiçoar a qualidade dos serviços prestados pela disponibilização do conhecimento útil em tempo oportuno, e com isso, fomentar na matriz curricular iniciativas que favoreçam a aplicação dos resultados de pesquisas aos cenários assistenciais ${ }^{37-40 .}$.

Aproximar pesquisa e ensino-serviço apresentou-se como ação complexa e desafiadora. A produção de investigações que possam estar articuladas às demandas dos contextos assistenciais e, por conseguinte, ao seu ensino, potencialmente devem ser amparadas por questões acadêmicoinstitucionais, como também por políticas científicas e tecnológicas de um país ${ }^{36-40}$. Um primeiro passo para a conciliação entre pesquisar-ensinar-cuidar foi observado pela imersão de docentes e pesquisadores nos serviços de saúde, de maneira materialdialógica, para ampliar o arcabouço daquilo que definem como questões de pesquisas ${ }^{40-42}$.

Nesta perspectiva, torna-se crucial o debate sobre novos conhecimentos que devem permear o saber epistemológico da Enfermagem e a prática docente. Estudos apontaram que esta prática apresenta lacunas na formação pedagógica específica, além do quê, há desconexão entre o trabalho docente e aspectos macrocontextuais, incluindo políticas que envolvam a formação dos recursos humanos em saúde, e um saber que valoriza principalmente a experiência prática $^{41,42}$. Uma pesquisa sobre como maximizar a aprendizagem da PBE entre os estudantes de Enfermagem evidenciou a transformação da atuação docente como principal viabilizadora do alcance desta condição, em associação com o desenvolvimento de macropolíticas que possam induzir e sustentar esta transformação ${ }^{43}$. Em contrapartida, políticas para a acreditação hospitalar têm induzido a integração ensino-serviço ${ }^{44}$. 
Por outro lado, pela perspectiva dos estudantes de Enfermagem, pesquisas identificaram que quando avaliam se os centros de formação têm possibilitado o ensino efetivo da PBE, referem lacuna expressiva desta abordagem na formação, e dificuldade em identificar evidências científicas que sejam relevantes para amparar mudanças práticas tanto por ausência de pesquisas como por pouca habilidade no julgamento crítico destas evidências.

As orientações para a utilização de recursos computacionais - identificação de descritores booleanos e aproximação às bases de dados da literatura científica de Enfermagem - uma das principais dificuldades mencionadas ${ }^{45-48}$. Contudo, a formação de enfermeiros reflexivos, com pensamento crítico, capazes de tomar decisões complexas e de basear-se em conhecimento pautado em evidências científicas é uma exigência cada vez mais premente frente as demandas atuais de saúde ${ }^{49,50}$.

Quanto às limitações desta revisão, entende-se que outros estudos primários poderiam ser incluídos por meio de buscas em outras bases de dados; em contrapartida, as principais bases de dados para a saúde (PubMed) e enfermagem (CINAHL) foram selecionadas, ainda que seja necessário destacar a escassez de investigações que tomem como ponto de partida as estratégias de ensino para superação dos desafios relacionados à PBE. Tal escassez é o principal dentre os aspectos que denotam a relevância do presente estudo, em especial quando se considera os países da América Latina e Caribe, sendo as publicações analisadas em sua maioria originárias de países do hemisfério norte.

Doravante, por a implementação da PBE caracterizar-se de forma desafiadora mundialmente, ainda se faz necessárias investigações sobre como tem sido desenvolvida a formação dos enfermeiros para essa prática. Em especial, identificar desenhos curriculares apoiadores a essa formação, orientados pelo pensamento crítico.
Ainda, torna-se imprescindível a discussão, no cenário contemporâneo, sobre metodologias que melhor apoiariam a construção crítico-reflexiva desse profissional, pautando-se na PBE. Por conseguinte, se faz necessário, o desenvolvimento de estudos prospectivos que avaliem o impacto da incorporação dessas estratégias na formação seja em disciplinas curriculares, ou em atividades que se aproximem do mundo do trabalho, e seus reflexos na qualidade da assistência por eles empreendidos.

\section{CONCLUSÃO}

Ao analisar o conhecimento produzido sobre estratégias para o ensino da PBE nos diferentes níveis de formação dos enfermeiros, pode-se perceber a identificação de estratégias no âmbito da mediação direta do processo ensino-aprendizagem, propiciadas pelas tecnologias de informação e comunicação, para reorientação da matriz educacional curricular.

Apesar das limitações desta revisão, em especial quanto ao número de estudos primários identificados, as evidências citadas podem ser úteis às discussões sobre o tema, bem como apoiar na orientação de alternativas de estratégias de ensino para o fortalecimento da formação do enfermeiro de práticas avançadas, ancoradas em conhecimento científico substanciado.

Sugere-se o empreendimento de pesquisas científicas futuras que abordem as dificuldades do corpo docente no ensino da PBE, as políticas existentes para apoio deste processo, a avaliação da integração-ensino serviço com vistas a fortalecer a PBE, e a divulgação de experiências de implementação dessas estratégias e dos resultados alcançados frente aos estudantes de enfermagem, em especial no âmbito na América Latina e Caribe, onde os estudos sobre o tema são incipientes.

\section{REFERÊNCIAS}

1. Melnyk BM, Gallagher-Ford L, FineoutOverholt E. The establishment of evidencebased practice competencies for practicing registered nurses and advanced practice 
nurses in real-world clinical settings: proficiencies to improve healthcare quality, reliability, patient outcomes and costs. Worldviews Evid Based Nurs. [Internet]. 2014 [citado em: 15 out 2016]; 11(1): 5-15. Disponível em: http://onlinelibrary.wiley.com/doi/10.1111/w vn.12021/epdf. Doi: 10.1111/wvn.12021.

2. Bick D, Chang YS. Implementation of evidence into practice: complex, multi-faceted and multi-layered. Rev Esc Enferm USP. [Internet]. 2014 [citado em: 22 Ago 2016]; 48(4):578-83. Disponível em: http://www.scielo.br/pdf/reeusp/v48n4/pt_0 080-6234-reeusp-48-04-578.pdf. http://dx.doi.org/10.1590/S0080623420140000400001.

3. Medina EU, Valenzuela CR, Pinto CT, Vidal MR. Enfermería basada en la evidencia: qué es, sus características y dilemas. Invest Educ Enferm. [internet]. 2010 [citado em: 22 Ago 2016]; 28(1):108-18. Disponível em: http://www.scielo.org.co/pdf/iee/v28n1/v28 n1a13.pdf.

4. Marziale MHP. El conocimiento modificando la práctica de la Enfermería [editorial]. Metas Enferm. [Internet]. 2016 [citado em: 22 Ago 2016]; 19(4):3. Disponível em: http://www.enfermeria21.com/revistas/meta s/articulo/80903/.

5. Edward KL. A model for increasing appreciation, accessibility and application of research in nursing. J Prof Nurs. 2015; 31(2):119-23.

6. Mccrae N. Whither nursing models? The value of nursing theory in the context of evidence-based practice and multidisciplinary health care. J Adv Nurs. [Internet]. 2012 [citado em: 22 Ago 2016]; 68(1):222-9. Disponível em: http://onlinelibrary.wiley.com/doi/10.1111/j. 1365-2648.2011.05821.x/abstract. Doi: 10.1111/j.1365-2648.2011.05821.x.

7. Parker JM. Knowledge production and reproduction: what are the implications for nursing practice? Nurse Educ Pract. 2009; 9(2):149-54.

8. Carvalho V. Linhas de pesquisa em enfermagem: destaques filosóficos e epistemológicos. Rev Bras Enferm. [Internet]. 2015 [citado em: $22 \mathrm{dez} 2016$ ]; 68(4):723-9. Disponível em: http://www.scielo.br/pdf/reben/v68n4/00347167-reben-68-04-0723.pdf. http://dx.doi.org/10.1590/0034-

7167.2015680421p.

9. Oliveira DC. Prioridades de pesquisa em enfermagem e as linhas de pesquisa: dando continuidade ao debate. Rev Enferm UERJ. [Internet]. 2014 [citado em: $22 \mathrm{dez}$ 2016]; 22(5):712-6.

Doi:

http://dx.doi.org/10.12957/reuerj.2014.1277

1.

10. Schaffer MA, Sandau KE, Diecrik L. Evidence-based practice models for organizational changes: overview and practical applications. J Adv Nurs. [Internet]. 2012 [citado em: 22 Dez 2016]; 69(5):1197-209. Disponível

em: http://onlinelibrary.wiley.com/doi/10.1111/j. 1365-2648.2012.06122.x/epdf.

Doi:

10.1111/j.1365-2648.2012.06122.x.

11. Zanetti ML. Prática avançada de enfermagem: estratégias para formação e construção do conhecimento [editorial]. Rev Latinoam Enferm. [Internet]. 2015 [citado em: 15 out 2016]; 23(5):779-80. Disponível em: http://www.scielo.br/pdf/rlae/v23n5/pt_010 4-1169-rlae-23-05-00779.pdf.

Doi: http://dx.doi.org/10.1590/0104-

1169.0000.2614.

12. Zanetti ML. Ensino e pesquisa na formação de profissionais do futuro [editorial]. Rev Latinoam Enferm. [Internet]. 2013 [citado em: 15 out 2016]; 21(3): [02 telas]. Diponível em: http://www.scielo.br/pdf/rlae/v21n3/pt_010 4-1169-rlae-21-03-0653.pdf. Doi: http://dx.doi.org/10.1590/S010411692013000300001.

13. Zug KE, Cassiani SHB, Pulcini J, Garcia AB, Aguirre-Boza F, Park J. Enfermagem de prática avançada na América Latina e no Caribe: regulação, educação e prática. Rev Latinoam Enferm. [Internet]. 2016 [citado em: 15 out 2016]; 24(e2807). Disponível em: http://www.scielo.br/pdf/rlae/v24/pt_0104-

1169-rlae-24-02807.pdf. Doi: 10.1590/15188345.1615.2807.

14. INP/APN Network, International Council of Nursing. ICN Nurse Practitioner [Internet]. Advanced Practice Nursing Network: Definition and Characteristics of the role. 2009.

15. Brehmer LCF, Ramos FRS. Integração ensino-serviço: implicações e papéis em vivências de cursos de graduação em Enfermagem. Rev Esc Enferm USP. [Internet]. 2014 [citado em: 22 dez 2016]; 48(1):119-26. Disponível 
http://www.scielo.br/pdf/reeusp/v48n1/pt_0 080-6234-reeusp-48-01-118.pdf.

Doi:

10.1590/S0080-623420140000100015.

16. Leonello MV, Oliveira MAC. Educação superior em Enfermagem: o processo de trabalho docente em diferentes contextos institucionais. Rev Esc Enferm USP. [Internet]. 2014 [citado em: $22 \mathrm{dez}$ 2016]; 48(6):1093102.

Disponível

em:

http://www.scielo.br/pdf/reeusp/v48n6/pt_0 080-6234-reeusp-48-06-1093.pdf.

Doi:

http://dx.doi.org/10.1590/S0080-

623420140000700018.

17. Mendes KDS, Silveira RCCP, Galvão CM. Revisão integrativa: método de pesquisa para a incorporação de evidências na saúde e na enfermagem. Texto \& Contexto Enferm. [Internet]. 2008 [citado em: $22 \mathrm{dez}$ 2016]; 17(4):758-64. Disponível em: http://www.scielo.br/pdf/tce/v17n4/18.pdf.

Doi: $\quad$ http://dx.doi.org/10.1590/S010407072008000400018.

18. Whittemore $\mathrm{R}$, Knafl $\mathrm{K}$. The integrative review: update methodology. J Adv Nurs. 2005; 52(5):546-53.

19. Soares CB, Hoga LAK, Peduzzi M, Sangaleti C, Yonekura T, Silva DRAD. Revisão integrativa: conceitos e métodos utilizados na enfermagem. Rev Esc Enferm USP. [Internet]. 2014 [citado em: 24 dez 2016]; 48(2):335-45. Disponível em:

http://www.scielo.br/pdf/reeusp/v48n2/pt_0 080-6234-reeusp-48-02-335.pdf.

http://dx.doi.org/10.1590/S0080-

6234201400002000020.

20. Santos CMC, Pimenta CAM, Nobre MRC. The Strategy PICO paragraph construction of the question of search and search for evidence. Rev Latinoam Enferm. [Internet]. 2007 [citado em: 15 out 2016]; 15(3):508-11. Disponível em: http://www.scielo.br/pdf/rlae/v15n3/pt_v15 n3a23.pdf.

http://dx.doi.org/10.1590/S0104-

11692007000300023.

21. Ursi, ES, Galvão CM. Prevenção de lesões de pele no perioperatório: revisão integrativa da literatura. Rev Latinoam Enferm. [Internet]. 2006 [citado em: 16 out 2016]; 14(1):124-31. Disponível

em:

http://www.scielo.br/pdf/rlae/v14n1/v14n1a 17.pdf. Doi: http://dx.doi.org/10.1590/S010411692006000100017.

22. Galvão CM, Sawada NO, Mendes IAC. A busca das melhores evidências. Rev Esc Enferm
USP. [Internet]. 2003 [citado em: $24 \mathrm{dez} 2016$ ]; 37(4):43-50. Disponível em: http://www.scielo.br/pdf/reeusp/v37n4/05.p df.

23. Stone C, Rowles CJ. Nursing students can help support evidence-based practice on clinical nursing units. J Nurs Manag. 2007; 15(3):367-70.

24. Adams S, MacCarthy AM. Evidence-based practice guidelines and school nursing. J Sch Nurs. 2007; 23(3):128-36.

25. McNett MM, Fusilero J, Mion LC. Implementing programs of nursing research and selecting doctoral nurse leaders. Nurse Leader. 2009; 7(1):42-6.

26. Gray MT. Research odssey: The evoluting of a research partnership between baccalaureate nursing students ans practicing nurses. Nurse Educ Today. 2010; 30(4):376-82.

27. Stiffler D, Cullen D. Evidence-based practice for nurse practitioner students: a competencybased teaching framework. J Prof Nurs. 2010; 26(5):272-77.

28. Larmon BH, Varner LW. The integration of evidence-based practice and research utilization in associate degree nursing curriculum: an approach at Mississippi University for Women. Teach Learn Nurs. 2011; 6(4):167-71.

29. Janke R, Pesut B, Erback L. Promoting information literacy through collaborative service learning in an undergraduate research course. Nurse Educ Today. 2012; 32(8):920-3. 30. Sortedahl C. Effect of online journal club on evidence-based practice knowledge, intent, and utilization in school nurses. Worldviews Evid Based Nurs. 2012; 9(2):117-25.

31. Zhang Q, Zeng T, Chen Y, Li X. Assisting undergraduate nursing students to learn evidence-based practice through self-directed learning and workshop strategies during clinical practicum. Nurse Educ Today. 2012; 23(5):570-5.

32. Buchholz SW, Budd GM, Courtney MR, Neiheisel MB, Hammersla M, Carlson ED. Preparing practice scholars: teaching knowledge application in the Doctor of Nursing Practice curriculum. J Am Assoc Nurse Pract. 2013; 25(9):473-80.

33. DeBruyn RR, Ochoa-Marín SC, Semenic S. Barriers and facilitators to evidence-based nursing in Colombia: perspectives of nurse educators, nurse researchers and graduate students. Invest Educ Enferm. [Internet]. 2014 
[citado em: 26 dez 2016]; 32(1):9-21. Disponível em: http://www.scielo.org.co/pdf/iee/v32n1/v32 n1a02.pdf. Doi: 53072014000100002.

34. Hunker DF, Gazza EA, Shellenbarger T. Evidence-based knowledge, skills, and attitudes for scholarly writing development across all levels of nursing education. J Prof Nurs. 2014; 30(4):341-6.

35. André B, Aune AG, Braend JA. Embedding evidence-based practice among nursing undergraduates: results from a pilot study. Nurse Educ Pract. 2016; 18:30-5.

36. Miranda DR. As duas vocações da Universidade: centralizar investigação e desenvolvimento; descentralizar conhecimento útil [editorial]. Rev Esc Enferm USP. [Internet]. 2015 [citado em: 16 dez 2016]; 49(Esp):1-6. Disponível em: http://www.revistas.usp.br/reeusp/article/vie w/109510/107983. Doi: 10.1590/S0080623420150000700001.

37. Mandelli M, Rigoli F. Application of research and information to human resources policies: regional goals for the Americas. Rev Esc Enferm USP. [Internet]. 2015 [citado em: $26 \mathrm{dez} 2016$ ]; 49(Esp2):154-59. Disponível em: http://www.scielo.br/pdf/reeusp/v49nspe2/e n_1980-220X-reeusp-49-spe2-0156.pdf. Doi: http://dx.doi.org/10.1590/S0080-

623420150000800022.

38. Oelke ND, Lima MADS, Acosta AM. Translação do conhecimento: traduzindo pesquisa para uso na prática e na formulação de políticas. Rev Gaúcha Enferm. [Internet]. 2015 [citado em: $26 \mathrm{dez}$ 2016]; 36(3):113-7. Disponível em: http://www.scielo.br/pdf/rgenf/v36n3/pt_19 83-1447-rgenf-36-03-00113.pdf. http://dx.doi.org/10.1590/1983-

1447.2015.03.55036

39. Mendes IAC, Trevizan MA, Leite JL, Godoy S, Ventura CAA. Políticas de produção de conhecimento em enfermagem. Rev Bras Enferm [Internet]. 2011 [citado em: $04 \mathrm{dez}$ 2016]; 64(3):415-22. Disponível em: http://www.scielo.br/pdf/reben/v64n3/v64n 3a02.pdf. http://dx.doi.org/10.1590/S003471672011000300002

40. Fortuna CM, Mishima SM, Matumoto S, Pereira MJB, Ogata MN. A pesquisa e a articulação ensino-serviço na consolidação do
Sistema Único de Saúde. Rev Esc Enferm USP. [Internet]. 2011 [citado em: 26 dez 2016]; 45(Esp2):1696-700. Disponível em: http://www.scielo.br/pdf/reeusp/v45nspe2/1 0.pdf. Doi: http://dx.doi.org/10.1590/S008062342011000800010.

41. Canever BP, Prado ML, Gomes DC, Jesus BH. Consciência de mundo epistemológica de docentes da área da saúde. Rev Gaúcha Enferm. [Internet]. 2016 [citado em: 26 dez 2016]; 37(3):e53811. Disponível em: http://www.scielo.br/pdf/rgenf/v37n3/01026933-rgenf-1983-144720160353811.pdf. Doi: http://dx.doi.org/10.1590/1983-

1447.2016.03.53811.

42. Lazzari DD, Martini JG, Busana JA. Teaching in higher education in nursing: an integrative literature review. Rev Gaúcha Enferm. [Internet]; 2015 [citado em: $26 \mathrm{dez}$ 2016]; 36(3):93-101. Disponível em: http://www.scielo.br/pdf/rgenf/v36n3/19831447-rgenf-36-03-00093.pdf.

Doi: http://dx.doi.org/10.1590/1983-

1447.2015.03.49670.

43. Christie J, Hamill C, Power J. How can we maximize nursing students' learning about research evidence and utilization in undergraduate, preregistration programmes? A discussion paper. J Adv Nurs. 2012; 68(12):2789-801.

44. Ribeiro HCTC, Campos LI, Manzo BF, Brito MJM, Alves M. Estudo das não conformidades no trabalho da enfermagem: evidências relevantes para melhoria da qualidade hospitalar. Aquichán [Internet]. 2014 [citado em: 26 dez 2016]; 14(4):582-93. Disponível em:

http://aquichan.unisabana.edu.co/index.php/a quichan/article/view/2934/3689. DOI: http://dx.doi.org/10.5294/aqui.2014.14.4.12 45. Adams S, Barron S. Use of evidence-based practice in school nursing: prevalence, associated variables, and perceived needs. Worldviews Evid Based Nurs. 2009; 6(1):1626.

46. Florin J, Ehrenberg A, Wallin L, Gustavsson P. Educational support for research utilization and capability beliefs regarding evidence-based practice skills: a national survey of senior nursing students. J Adv Nurs. 2012; 68(4):88897.

47. Brooke J, Hvalič-Touzery S, Skela-Savič B. Student nurse perceptions on evidence-based practice and research: an exploratory research 
study involving students from the University of Greenwich, England and the Faculty of Health Care Jesenice, Slovenia. Nurse Educ Today. 2015; 35(7):e6-e11.

48. Adams S. Use of evidence-based practice in school nursing: survey of school nurses at a national conference. J Sch Nurs [Internet]. 2009 [citado em: 26 dez 2016]; 25(4):302-13. Disponível em: http://journals.sagepub.com/doi/pdf/10.1177 /1059840509335008.

DOI: $10.1177 / 1059840509335008$

49. Oliveira LBO, Díaz LJR, Carbogim FC, Rodrigues ABR, Püschel VAA. Efetividade das estratégias de ensino no desenvolvimento do pensamento crítico de graduandos de Enfermagem: uma metanálise. Rev Esc Enferm USP. [Internet]. 2016 [citado em: $26 \mathrm{dez} 2016$ ]; 50(2):355-64. Disponível em: http://www.scielo.br/pdf/reeusp/v50n2/pt_0 080-6234-reeusp-50-02-0355.pdf. DOI: http://dx.doi.org/10.1590/S0080623420160000200023

50. Erdmann AL, Pagliuca LMF. O conhecimento em enfermagem: da representação de área ao comitê assessor de enfermagem no CNPq. Rev Bras Enferm. [Internet]. 2013 [citado em: $26 \mathrm{dez}$ 2016]; 66(Esp):51-9. Disponível em: http://www.scielo.br/pdf/reben/v66nspe/v66 nspea07.pdf. DOI: http://dx.doi.org/10.1590/S003471672013000700007

CONTRIBUIÇÕES
Fernanda Carolina Camargo atuou na
concepção, análise dos dados, redação e
revisão crítica. Helena Hemiko Iwamoto,
Gilberto de Araújo Pereira e Regiane
Máximo de Souza contribuíram na revisão
crítica. Luan Augusto Alves Garcia
participou na redação e revisão crítica.
Damiana Aparecida Trindade Monteiro
contribuiu na coleta dos dados e revisão
crítica. Sabrina Karla de Souza Cubas e
Gabriela França Rosinha participaram na
coleta dos dados e redação.

Como citar este artigo (Vancouver)

Camargo FC, Iwamoto HH, Pereira GA, Souza RM, Garcia LAA, Monteiro DAT et al. Estratégias para o ensino da prática baseada em evidências na formação de enfermeiros: revisão integrativa. REFACS [Internet]. 2018 [citado em inserir dia, mês e ano de acesso]; 6(Supl. 1):363-374. Disponível em: inserir link de acesso. DOI: inserir link do DOI.

\section{Como citar este artigo (ABNT)}

CAMARGO, F. C. C. et al. Estratégias para o ensino da prática baseada em evidências na formação de enfermeiros: revisão integrativa. REFACS, Uberaba (MG), v. 6, n. 1, p. 363-374, 2018. Supl. 1. Disponível em: inserir link de acesso. Acesso em: inserir dia, mês e ano de acesso. DOI: inserir link do DOI.

\section{Como citar este artigo (APA)}

Camargo, F. C., Iwamoto, H. H., Pereira, G. A., Souza, R. M., Garcia, L. A. A. \& Monteiro, D. A. T. et al. Estratégias para o ensino da prática baseada em evidências na formação de enfermeiros: revisão integrativa. REFACS, 6(1), 363-374. Recuperado de: inserir link de acesso em inserir dia, mês e ano de acesso. DOI: inserir link do DOI. 